III.

Aus der Professor Oppenheimschen Polizlinik fïr Nervenkranke.

\title{
Über das kombinierte Vorkommen von Myasthenie und Basedowscher Krankheit, nebst Bemerkungen über die okulären Symptome der Myasthenie.
}

\author{
Von \\ 1)r. med. LOESER, \\ Berlin.
}

Die Kombination \% weier Nervenkrankheiten, vnn denen jede cinzelne bemerkenswerte Veränderungen am dugenapparat herbei\%uführen vermag und oftmals gerade hier am markantesten in die Erscheinung tritt, hat naturgemäss auch für den Augenart ein erhebliches Interesse. Das veranlasst mich, über zwei Beobachtungen zu berichten, die ich in den let\%ten Monaten machen konnte, die eine in der Nervenpoliklinik des Herrn Prof. Oppenhein, die andere in meiner eigenen Praxis. Die Krankengeschichten sind, im Aus\%ug mitgeteilt, folgende:

Fall 1. Die 21jăhrige Mrbeiterin Lene K. suchte am 15. 11!. 1904 die Poliklinik auf und gab an, dass iha am 9. Ill. im Latufe von \% wiolf Stunden das linke obere Augenlid \%ugefallen sei, ohne irgendwelche Begleiterscheinungen, wie Kopfschmer\%en, Ubelkeit oder Eibrechen. Im Oktober 1903 habe das Lid sehon ainmal 8 'lage lang heruntergebangen: es sei spontane Rückbildung cingetreten. Patientin klagt fernor über allgemein-nervöse Beschwerden, kommt leicht ins Weinen, ist sehr empfindlich, leicht erregt, \%ittert viel.

Am linken $\triangle$ uge besteht eine fast komplete Ptosis mit sckundïrer Anspannung des M. frontalis: auch durch maximale Kontraktion des linken Frontalis wird das Oberlid nur wenig gehoben. Das Lid ist ohne Fältelung und zeigt ein exquisit myasthenisches Verhalten. Wenn nimlich Patientin das Auge einige Minuten lang geschlossen gehalten hat, kamn sie das Oberlid beim ersten Versuch nahe\%u vollkommen heben, sie kann es aber nicht dauernd hoch halten; sehr bald fängt das Lid :tu, wieder herunterzusinken, bis schliesslich eine fast totale P'tosis eingetreten ist.

Aul Belragen gibt Patientin auchan, dass sie morgens das a uge besser aufmachen kann, auch wenn sie nachmittags geschlafen habe. Der Augenschluss ist beiderseits ganz kraftlos; auch reflektoriseh ist knin genügender Augenschluss \% er\%ielen. Die Konvergen\% ist gut: rechts besteht eive Andeutung von Graefes Symptom. Beiderseits lixophthalmus, rechts $>$ als links; dic Augenbewegungen sind irei, der dugenlintergrund etc. normal. Pupillen gleich weit, reagieren prompt. Der Isippenschluss ist kräftig; es tritt keine Ermüdung beim 
Sprechen ein. In den Händen ein unbestimmter, rascher, vibrierender Tremor. Puls augenblicklich 140. Starke Abmagerung: geringe, nicht pulsierende Struma. Cor normal. Die Tachykardic dauert wäbrend der Untersuchung an.

In anderen Muskelgebieten keine pathologischen Ermüdungserscheinungen nachweisbar. Sehnenphänomene stark.

16. III. Es besteht heute Strab. diverg. Die Insufficienz der M. recti interni ist ganzausgesprochen. Auchim rechten Levator hesteht geringe Érmüdbarkeit.

Bei faradischer Rei\%ung des M. deltoideus und Supinator longus lässt sich deutliche myasthenische Reaktion nachweisen. Mit mïssig starken Strömen eriolgt \%unächst eine krāftige liontraktion des Deltoides; līsst man diesen Strom datuernd einwirken, so erbält man allmählich an Stelle der mit einer Lokomotion verbundenen Kontraktion ein fibrilläres Wogen der einzelnen Muskelbūndel, bis schliesslich auch dieses ausbleibt und der Muskel auf den faradischen Rei\% überhaupt nicht mehr reagiert. Auch bei rasch hintercinander immer wieder unterbrochenen faradischen Reizungen wird die Kontraktion des Muskels sehr rasch (nach 1(i-15 Rei\%ungen) schwiacher und rersagt schliesslich gitn\%. Auch im linken Cucullaris und Supinator longus ist myasthenische Reaktion nachweisbar. Der Puls bleibt auch während der heutigen Untersuchung andatuernd beschleunigt und klein.

Fall 2. Die 3ajjährige Patientin Fratu f. ist seit dem Juhre 189? krank. Sie litt damals an starkem Her\%klopfen, allgemeiner Schwäche und Mattigkeit und nahm in 4 Wochen 20 Pfund ab. Es stellte sich damals eine Vergrösserung der Schilddrüse ein, und die Augen traten aus ihren Hïhlen bervor. Sie hatte ferner über Durchfälle, Schweisse und Zittern z.u kligen. Laingerer Aufentbilt in der Prof. Mendelschen Klinik wegen "Worlus Basedowii".

In der liolgezeit fingen die $A$ ugenlider an, etwats herunterzuhängen, und seit 8 Jahren kann sic die $\lambda$ ugen nicht ganz ïlinen. Ermüdungsgefühl beim Sprechen, gelegentlich Heiserkeit nach lingerem Sprechen. Erschwerung des Kauens und Schluckens; auch beim Gehen leichto Ermüdbarkeit. Der Krank. heits\%ustand ist im gan\%en wechselnd; an schlechten Tagen kann sie nur j-10 Minuten gehen. Auch in den Händen bei der Tätigkeit allmählich sich einstellende Schwäche, so dass ihr die Gegenstände aus den Höinden fillen. Bisweilen

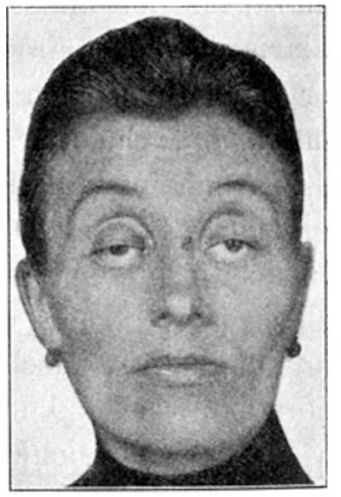
Schwierigkoit, don Kopf vornüber 211 halten. Sie lässt dann dis Nackenmuskeln eine Weile erschlaffen und ist wieder imstande, den Kopf zu halten. Die Strumi ist in den let\%en Jahren erheblich \%urückgegangen, auch das Her\%klopfen tritt nur noch bei Erregungen ein.

Beide Augenlider hägen schlaff herab und können nur durch starke dospannung der liroutales soweit gehohen werden, dass etwa die kleinere Halfte der Cornea in der Lidspalte frei \% T Tage tritt. Der gan\%e Gesichtsausdruck erhält dadurch einen eigenatrtigen, schläfrigen, müden Charakter (cf. Abhildung). De d ugenschluss ist im gan\%en schwächer als normal: bat siedie Augeneine Weile \% unekafien, so tritt besonders links nochein merklicher Nachlass der liraft ein. Keine Schwäche der lionvergen\%. Die Augenbewegungen sind frei. Überhaupt ist der Augenapparat, von geringer Nyopie algesehen, sonst vollkommen normal. Rechts wurde mehrmals $\lambda$ rterienpuls beobachtet. 
Nach mehrmaligem Vorwärtsneigen des liopfes tritt eine Schwäche ein, so dass der Kopf auch gegen leichten Widerstand nicht mehr nach rorn gebeugt werden kann.

Dasselbe bei Rückwärtsneigung des Koples.

Die Schilddrüse ist nur wenig vergrössert. Patientin ist im allgemeinen sehr miger. Bei 20 maligem Händedruck lässt die Kiraft erheblich nach.

Die myasthenisch-elektrische Reaktion ist deutlich im linken Sternocleido-mastoideus und im Deltoideus nachweisbar.

Puls augenblicklich 1()4. Cor normal.

Aus der mir durch dic lireundlichkeit des Herrn Kollegen lirit\% Mendel zur Verfügung gestellten Krankengeschichte aus dem Jahre 1892 gelut hervor, dass die Patientin damals rom 23. V. bis 28. VI. in klinischer Behandlung gestanden hat. Die Diagnose lautete: Morbus Basedowii und Hysterie.

Die in diesen beiden Fällen gestellte Diagnose einer Ḱombination von Morbus Basedowii mit Myasthenie bedarf eigentlich kaum der Begründlung.

Im Falle I fanden wir Exophthalmus, Andeutung von Graefes Symptom, Tachykardie, vibrierenden 'l'remor', leichte Struma, starke Abmagerung, allgemein-nervöse Beschwerden, also einen die Diagnose Morb. Based. hinreichend sichernden Symptomenkomplex. Daneben konnten wir den myasthenischen Charakter der linksseitigen Ptosis und ler doppelseitigen Orbicularisschwäche einwandsfrei feststellen und durch den Nachweis der myasthenischen Reaktion im linken Deltoideus, Supinator longus und Cucullaris die Diagnose "Myasthenie" bestaitigen.

Auch im Fall II machte die Diagnose "Myasthenie" keinerlei Schwierigkeiten, da das funktionell myasthenische Verhalten der Muskelstörung in einer gan\%en Reihe von Muskelgruppen (Orbicularis oculi, Kopf- und Nackenmuskulatur etc.) nachweishar war und auch die typische Verïnderung der olektrischen Frregharkit gefunden werlen konnte.

Nicht so sicher hätte dagegen hier die Frage be\%. Ier Basedowschen Krankheit entschieden werden können, wenn nicht der ärtliche Krankheitshericht aus dem Jahre $1892 \%$ Hülfe gekommen wäre. Denn die heute noch liestehenden auf einen Basedow zu beziehenden Merkmale - die 'lachykardie, die Struma und der Fxophthalmus - wïrden nicht ausreichen, die Diagnose zu stützen, da erstens die 'lachykardie nicht selır erheblich, zweitens die Struma nur selır geringfügig und growiss nicht eindeutig ist, drittens der Exophthalmus differential-diarrnostisch hier kaum in betracht kommen kann. (Auch unkomplizierte Myasthenien zeigen nämlich gelegentlich Prominen\% der Bulbi.) Auf Grund des klinischen Berichts und der anamnestischen Angaben unterliegt es aber keinem \%weifel, dass wir as auch 
hier neben der Myasthenie mit einer Forme fruste des Morb. Based. zu tun haben. Lnd darin liegt gerade das besondere Interesse dieses Falles, der uns zeigt, wie auf dem Boden eines Morb. Basedowii im Laufe der Jahre unter Rückbildung der Basedow-Symptome eine typische Myasthenie sich entwickelt hat. Er gibt für die Annalıme Oppenlieims '), wonach es bei einem kombinierten Vorkommen dieser Krankheiten gewöhnlich die Basedowsche Krankheit ist, die die Disposition zur Erkrankung an Myasthenic schafft, ein bemerkenswertos Beispiel.

Auf das Wesen dieses \%weifellos bestehenden inneren \%usammenhanges der beiden Nervenkrankheiten gehe ich nicht weiter ein. Dafür möchte ich noch den Auszug aus der Krankengeschichte eines dritten, seit \%wei Jahren ${ }^{2}$ ) in Beobachtung von Herrn Prof. Oppenheim befindlichen Falles von unkomplizierter Myasthenie anfügen, der eine Reihe hemerkenswerter okuliirer Symptome darbietet.

Die 25jährige Näberin $\triangle$ / rine N., die am 14. 11. 1902 \%um ersten Male die Puliklinik aufsuchte, hat seit cal. einem halben Jabre bemerkt, duss sie ibr rechtex Oberlid, besonders gegendbend, nicht mehr gut hochleben küuns, "nd bei Blickbewegungen nach rechtsbisweilen doppelt sieht. Sie habe hereits die Augenklinik von Dr. $X$. aufgesucht, wo sie mit einer. Schmierkur behandelt worden sei. bin unmittelbarer Einfluss dieser Behandlung war nicht \%u ermitteln, sie habe vielmehr die lílinik in einem erheblich verschlechterten Allgemeinzustande verlassen: erst wach mehreren Wochen nach Beendigung ler Kur trat geringe Besserung ein.

Die Lähmung halte sich ohne Kopfschmerzen oder sonstige Nebenerscheinungen entwickelt. Gelegentlich sei es auch rorgekommen, dass sie abends das andere Lid nicht gut habe heben können. A m Morgen sei die Beweglichkeitsstorung stets geringer. In den Armen und Beinen verspürt sie keinerlei Müdigkeit, anch beim Kianen, Sehlingen, Sprechen keine Beschwerden.

Es besteht rechts eine deutliche Ptosis mit sekundïler in. spannung des M. frontalis. Im Laule der Untersuchung wird die Ptosis rechts stäker und a uch das linke()l,erlid sinkt einwenig herab. Nach längerem Oifenhalten der lngen wird die Ptosis rechts total, und erst nach einer langeren liuhepause rersctwindet sie wieder.

Boim Blick nach rechtsbleibt let rechte Bullus dentlich z. uri ck; auch die übrigen lindstellungen werden nur mühsam erreicht, unter nystagmusartigen Zuckungen. Sonst ist der Augenbefund (Myopie) normal. Die Sprache reigt koine Ermüdung. In den Armen und Beinen sind keine myasthenischen Erscheinungen nachweisbar.

Doutliche myasthenische Reaktion im linken Deltoideus, die allerdings etwas spät cintritt.

1) Die inyasthenische Paralyse. Berlin 1901. S. Kóager. p. 135.

2) Dieser liall wird im \%usammeuhang mit den äbrigen in den let\%ten Jahren beohachteten Myasthenien ausführlich von anclerer Scite mitgeteilt werden. 
16. II. Heute lässt sich auch im rechten Rect. sup. Ermüdung feststellen, desgleichen im Orbicularis oculi, der nach mehrmaligem lirüftigen Lidschluss erheblich geschwïcht ist. Auch beute sichere My.R. im linken Deltoideus. Sehr auffällig ist die starke Dyspnoe bei geringer Muskelanstrengung und im ganzen normaler Pulsfrequenz.

27. IV. 1904. Die Pat. gibt an, dass sich der Zustand ihrer Augen wesentlich verschlechtert habe. Das linke Oberlid kïnne sie schon seit last 1/2 Jahre gar nicht mehr heben und auch das rechte sei viel schwächer geworden. Dic Untersuchung ergab: Das rechte Oberlid \%eigt cin typisch myasthenisches Verhalten (cf. Fall 1). Augenschluss gan\% kraftlos. Bulbus in leichter Divergenz. Die Bewegung nach oben und innen ist n:Lezu aufgehoben, nach aussen und unten erheblich eingeschränkt. Nach längerem ruhigen Geschlossenhalten des Auges kann eine deutliche Adduktionsbewegung ausgeführt werden. Die Pupillenreaktion ist prompt erbalten. J.inks besteht komplete Ptosis. Nur durch starke Anspannung cies l'rontalis gelingt es, die Lidspalte ein wenig zu óffnen. Alle übrigen Bulbusbewegungen feblen fast vollkommen; nur noch geringe Trochleariswirkung nachweisbar. Dabei ist die Pupillenreaktion in normaler Weise vorhanden.

An der Diagnose nMyasthenie" kann in diesem Falle bei dem Nachweis der typischen funktionellen Muskelstörung (in den Levatoren der Oberlider in den Orbiculares oculi) die hier gan\% besonders in die Augen springt, und bei dem Verhalten der elektrischen Erregharkeit im Deltoideus nicht der geringste Zweifel sein.

Von besonderem Interesse ist hier, dass sich aus der anfangs passageren Lrmüdbarkeit und Muskelschwäche allmählich eine stationare Ophthalmoplegie herausgebildet hat, deren Fntstehung und Natur man heute, soweit sie das linke Auge betrifit, ohne weiteres nicht erkennen könnte.

Was nun die myasthenischen Frscheinungen der mitgeteilten Fälle betrifft, so konnten wir übereinstimmend feststellen, dass in allen drei die okulären symptome es waren, die die ersten in die Erscheinung tretenden Krankheits\%eichen überhaupt bildeten. Dieser Beginn des Iseidens ist sehr gewöhnlich, und Oppenheim ${ }^{1}$ ) gibt \%. B. in seiner hekannten Monographie an, dass in 15 von 51 Fällen zuerst der Augenmuskelapparat von der Störung befallen würde. Die Kenntnis des myasthenischen symptomenkomplexes ist also für den $\mathrm{d}$ ugenar\%t von grosser Wichtigkeit. Demgegenüber ist es auffällig, dass in den bekannteren Lehrbüchern der Augenheilkunde der Myasthenie als ätiologischen Faktors der Augenmuskelstïrungen gar nicht oder nur beiläufig Erwähnung getan und auf die charakteristischen Eigenschaften der Bewegungsanomalie nirgends hingewiesen ist. Eine kurze Besprechung der Sympto-

3) Die myasthenische Paralyse. Berlin 1501. Verlag von S. Karger. 
matologie der Myasthenie, soweit sie den Augenapparant betrifft, dürfte deshall, an dieser Stelle nicht unangebracht' ${ }^{1}$ ) sein, um so mehr, als die mitgeteilten Krankengeschichten eine treffliche Illustration dazu abgeben.

Die hauptsächlichste Rolle spielt dic ein-oder doppelseitige Ptosis, indes treten sehr oft auch Isähmungserscheinungen der äusseren Bulbusmuskeln \%u Tage, während die Binnenmuskulatur des Auges immer verschont bleibt. Recht oft nimmt auch der rom Facialis versorgte Schliessmuskel des Auges an der Erkrankung teil, sodass eine deutlich nachweisbare Schwäche desselben, inschwereren Fällen auch ein ausgesprochener Lagophthalmus bestehen kann. "Seltener wurden nehen den Zeichen der Funktionsbeschränkung auch Reizphänomene in Form des I,idzitterns, derNaictitatio, des Blepharoclonus walırgenommen “"). Der Augenhintergrund wurde stets normal befunden.

Diese kurze Aufrählung der okulïren Symptome der Myasthenie reigt, dass in ilurer Lokalisattion und in ihrer Kombination an sich nicht immer etwas Charakteristisches, der Krankkeit Eigentümliches \%u finden ist. Nur ron derdoppelseitigen Ptosis, die allerdings in $78, \overline{0} \mathrm{pCt}$. der bis jet\%t in der loiteratur bekannten Fïlle vorkommt ${ }^{3}$, kimn vielleicht gesagt werlen, dass sie sonst kaum hei einer anderen Affektion beobachtet wird und demgemïss bis \%u einem gewissen Gra!le als Charakteristikum für diese Krankheit an\%usprechen ist. Findet sich, was sehr oft der Fall ist, neben ihr noch eine Schwäche der Gesichtsmuskulatur, so erhält dadurch und durch die sekundäre Anspannung der $\mathbf{M}$. frontales der gran\%e Gesichtsausdruck etwas so Clarakteristisches (ct. Fig. 1), dass man eine prima vista Diagnose stellen kann, wie es \%. B. bei meinem fiall 2 geschah. Auch das konstante Freibleiben des Sphincter pupillace und M. ciliaris im Verein mit multiplen Jähmungen der ïusseren Bulbusmuskeln gibt gelegent.lich einen Finger\%eig für die Diagnose des Grundleidens. (ct. Fall 3). Mit Sicherheit kann diese aber erst clurch den Nachweis des

1) Ausser meiner persünlichen Erfahrung, dass ich als Assistent unter vielen Tausenden Augenkranker keinen einzigen fall ron mysthenischer Augenmuskelläbmung gesehen bezw. diagnosti\%iert hatte, wälirend ich in den let\%ten Jahren in der Prof. Oppenheimschen Poliklinik eine ganze Reihe derartiger, zum grossen Teil aus der Kgl. Universitïtsaugenklinik (Geh. Rat s. Michel) zur Untersuchung überwiesener Fälle beobachten konnte, machte ich auch bei \%ahlreichen Fachgenossen die Feststellung, dass ihnen das Symptomenbild der Mysthevic nur wenig bekannt war.

2) Oppen heim, I. c. p. 73.

3) Wilbrand-Saenger, Die Neurologie des Auges. Bd. 1. p. 220. 
eigenartigen qualitativen Verhaltens der Muskelstörung gestellt werden, deren vorherrschendes und charaliteristisches Zeichen die abnorme Lirschüpfbarkeit der Muskulitur, "die Myasthenie" ist, sodalss also ein \%unächst gut funktionierender Muskel durch jede Kontraktion geschwächt wird, bis schliesslich mit immer erneuter Inanspruchnahme ein völliges Versagen erfolgt.

So wird es verstindlich, dass so oft die Augemmuskulatur die doch ausser im Schlate in fortwährender Aktion ist, die Reihe der uyasthenischen Symptome eriffnet und gerade an dieser dauernde, wenn aluch in ihrer Erscheinungsform wechselnde Lähmungserscheinungen vorkommen. Auch stimmt damit die oft zu hörende Angabe des Patienten überein, dass ,morgens nach dem Erwachen die Funktionsstörung meistens am wenigsten ausgesprochen, ja völlig zurückgetreten sein kann, wïhrend sie in Laufe des Tages mehr und mehr \%unimmt"1).

Das Wesen der myasthenischen funktionsstörung lässt sich in vielen Fällen, gerade an Augenmuskelapparat, schlagend nachweisen: wir sehen, wie das Oberlid \%unächst galn\% gut gehoben wird; mit der zunehunenden Folge des Lidschlags nimmt die Funktionsfähigkeit ab, bis schliesslich der Innervationsimpuls ohne jeden Lffekt bleibt; das Lid hängt, wie ein völlig gelïhmtes, schlaff herab, die Lidspalte wird nur lurch Frontalis-Wirkung geöffnet. (cf. Fall 1 und 3.) Auch in anderen Bulbusmuskeln lassen sich gelegentlich Ermüdungserscheinungen nachweisen (cf. Fall 3. Rectus internus dext.), für die unter anderem die anamnestische Angabe von der Flüchtigkeit des Doppelsehens beweisend ist.

Aus unserer Schilderung geht schon hervor, dass die Lähmungserscheinungen iiusserst wechselnd sein können, sowohl an Intensität wie Extensitait. Es liegen Beobachtungen vor, wo an einem Tage innerhalb weniger Stunden bemerkenswerte Schwankungen vorgekommen sind. A uf der anderen Seite ist $\% u$ betonen, dass auch der eine oder andere Muskel konstant und dauernd gelähmt bleiben kann. Das yeigt uns unser Fall 3, wo sich am linken Auge allmählich eine stationire Lühmung entwickelt hat, die schon seit langem in unverinderlicher Form fortbesteht. Eine solche etwa mit Strümpell nur als die "Konsequen\% der Myasthenie “, einen „konstanten Lrmündungšustand" \%u betrachten, scheint wenig plausibel, sie spricht vielmelır für die Anschaung

1) Oppentueim, I. .. p. 6i. 
Oppenheims, lass auch eine ec h te Muskelschwäiche und lıähmung vorkommen kann.

Set\%t urs schon eine genaue Wïrdigung der okulären Symptome oft in den Stand, die Diagnose "Myasthenie" mit einiger Sicherheit \%u stellen, so bleiben doch auch vicle Fälle übrig, wo das der myasthenischen Funktionsstörung Eigentümliche zunächst nicht nachweishar ist. Oft geben hier die anamnestischen Angaben, \%. B. rasches Heiserwerden der Stimme, Beschwerden beim Kauen und Schlingen, Schwäche der Nacken- und Kopfmuskulatur, schnelle Ermüdbarkeit der Gliedmassen etc. etc. einen wertvollen Finger\%eig; die Diagnose kann aber erst durch cine sperialistisch-neurologische Untersuchung, die natürlich in allen Fällen vorgenommen werden muss, speriell durch den Nachweis der "myasthenischen Reaktion" gesichert werden.

Auf alle diese Dinge, die vielgestaltige und wechselnde Symptomatologie, gehe ich selbstredend an dieser Stelle nicht näher ein. Es kam mir nur darauf an, auf Grund der mitgeteilten Beobachtungeu einmal auf die okulairen Symptome der Myasthenie und kur\% auf die Merkmalo lin\%uweisen, die schon den Augenar»t \%ur richtigen Diagnose führen können. Und dass die frühe Frkenntnis dieses Leidens von der grössten praktischen Bedeutung ist, lehren die gar nicht so vereinzelten Erfalırungen, wo derartigre: Fälle in Ermangelung einer greiflaren Ätiologie zunächst mit Scluwit\%en, fodkali, Schmierkur oder einer Kombination dieser beliebten Heilfaktoren behandelt wurden (cf. Fall 3), Massnahmen, die bei der Natur des Grundleidens wegen ihres schwächenden Einflusses grerale kontrandiriert sind und gelegentlich eine erhebliche Verschlimmerung des Allgemeinzustancles im Gefolge hat,ten, durch plötzliche Insuffiøienz der Atemmuskulatur wohl auch einmal den Exitus letalis herbei\%uführen vermögen.

Herm Professon Oppenheim, meinem hochverehrten Lehror, silge ich für die Erlaubnis \%ur Veröffentlichung der aus seines Poliklinik stammenden Fïlle meinen ergebensten Dank. 